\title{
Embarazo en condición juvenil: contexto histórico en Colombia y trayectoria en ciudad fronteriza
}

\section{Pregnancy in juvenile condition: historical context in Colombia and trajectory in the border city}

\author{
Doris Amparo Parada Rico ${ }^{1}$
}

1. Universidad Francisco de Paula Santander. Cúcuta, Colombia. Correo: dorisparada@ufps.edu.co - https://orcid.org/0000-0003-4713-6688

Tipología: Artículo de revisión

Para citar este artículo: Parada-Rico DA. Embarazo en condición juvenil: contexto histórico en Colombia y trayectoria en ciudad fronteriza. Duazary. 2020 julio - septiembre; 17(3): 86- 100. Doi: http://dx.doi.org/10.21676/2389783X.3316

Recibido en enero 17 de 2019

Aceptado en noviembre 08 de 2019

Publicado en línea en abril 18 de 2020

\section{RESUMEN}

Palabras clave: adolescente; juventud; salud sexual; características culturales.

Al hacer referencia al embarazo en adolescentes o jóvenes, de una parte, implica todo un abordaje acerca del riesgo en la salud estimado para la madre y su hijo por nacer; y de otra, la connotación dada al hecho de maternar o paternar dentro de una temporalidad y trayectoria del curso de vida asignada socialmente para estudiar, laborar o dedicarse simplemente al cuidado de sí. El objetivo del actual trabajo es analizar el panorama histórico del embarazo en condición juvenil en Colombia y su trayectoria en el municipio de Cúcuta durante el periodo de 2012 a 2017. La metodología utilizada fue la búsqueda y revisión sistemática de investigaciones, documentos históricos, normativas, Actas de las mesas intersectoriales municipal y departamental de "prevención de embarazo en adolescentes" alrededor del tema. Dentro de los resultados hallados, se observa una perspectiva de riesgo marcada, construida históricamente, así como una tendencia al aumento de embarazos en menores de 15 años. Conclusiones: se hace necesario establecer nuevas vías que permitan promover en conjunto con adolescentes y jóvenes, la toma de decisiones basada en sus derechos sexuales y reproductivos, teniendo en cuenta además el contexto en el que se inscriben determinantes sociales asociados.

Keywords: adolescent; youth; sexual health; cultural characteristics.

\section{ABSTRACT}

When referring to pregnancy in adolescents or young people, on the one hand, it implies a whole approach to the estimated health risk for the mother and her unborn child; and on the other, the connotation given to the fact of maternating or paternalizing within a temporality and trajectory of the socially assigned course of life to study, work or simply dedicate itself to caring for oneself. The objective of the current work is to analyze the historical panorama of pregnancy in youth condition in Colombia and its trajectory in the municipality of Cúcuta during the period from 2012 to 2017. The methodology used was the search and systematic review of investigations, Historical, normative documents, Minutes of the municipal and departmental intersectoral tables of "teenage pregnancy prevention" around the subject. Among the results found, there is a marked risk perspective, historically constructed, as well as a tendency to increase pregnancies in children under 15 years. Conclusions: it is necessary to establish new ways to promote, together with adolescents and young people, decision-making based on their sexual and reproductive rights, also taking into account the context in which associated social determinants are registered.
\end{abstract}




\section{INTRODUCCIÓN}

Para iniciar este análisis, se hace necesario referir que al hacer mención al embarazo en condición juvenil, este se vincula inmediatamente a lo definido como embarazo adolescente, el cual se concibe en el "protocolo de atención a la menor de 15 años embarazada" diseñado por el Ministerio de Salud y Protección Social y el Fondo de Poblaciones Unidas (UNFPA) en el año 2014, como aquel... no planeado ni esperado que ocurre en una mujer o en una pareja que está(n) económicamente dependiendo de otros..., viéndose forzados a interrumpir su proceso de desarrollo humano (escolarización, empleo, planes de vida)...1; no obstante debido a la diferencia de contextos, culturas y políticas, así como de los rangos etarios planteados para definir adolescencia o juventud, se requiere ahondar un poco más en el tema, entendiendo de una parte, la condición juvenil como el "conjunto multidimensional de formas particulares diferenciadas y culturalmente acordadas" en la que se encuentran los sujetos "... inmersos en relaciones de clase, de edad, de género, étnicas" ${ }^{2-4}$, y de otra la relevancia de encarar este análisis desde una triple complejidad: contextual: espacial e históricamente situado; relacional: conflictos y consensos; y heterogénea: diversidad y desigualdad" ${ }^{5}$.

Con respecto a la diferencia de rangos etarios que definen esta población, existen clasificaciones que traslapan adolescencia y juventud. De una parte, la Organización Mundial de la Salud $(\mathrm{OMS})^{6}$, ha establecido la adolescencia como el periodo entre los 10 a 19 años; en Colombia, a partir de la Ley 1098 de $2006^{7}$ (código de Infancia y la Adolescencia), y la Ley 1622 de $2013^{8}$ (Estatuto de Ciudadanía Juvenil), se plantean edades que entrecruzan los rangos estimados para la adolescencia y juventud, de tal manera que no hay una identificación exacta por etapas. Igualmente se evidencia la amplitud de rangos de edad en los que se presentan embarazos en condición juvenil, lo cual permite analizar heterogeneidades en los distintos contextos.
De esta forma, puede resultar que acercándose a la noción presentada como condición juvenil, emerjan comprensiones respecto a la presencia de embarazos en este grupo de sujetos, que permitan evaluar el panorama de este fenómeno de manera contextualizada tanto geográfica como históricamente, relacional y alejada de marcos condicionados y determinados por los discursos que tienden a generalizar y limitar el conocimiento de las diversas formas de ser joven, y sobre todo, que impiden valorar aspectos de la multiculturalidad existente en cada lugar en particular.

Lo anterior implica valorar resultados de estudios en los que se aborda, por ejemplo el riesgo en la salud de las mujeres embarazadas en condición juvenil, así como de sus hijos por nacer, dentro de los que se halla que a menor edad de la madre, existe mayor riesgo de prematurez y de peso bajo para la edad gestacional $^{9-10}$. No obstante, se evidencian también trabajos muy amplios realizados en contextos diversos, como el de Althabe et $a 1^{11}$, estudio de investigación prospectiva en el que participaron mujeres menores de 24 años; realizado en 6 países de ingresos medios-bajos (Kenia, Zambia, India, Pakistán, Guatemala y Argentina), evidenciándose que los embarazos en menores de 15 años, no poseen mayor riesgo de resultados adversos maternos, pero si se relaciona su resultado con el contexto en el que habita la madre; siendo muy parecidos los hallazgos en América Latina y África Subsahariana.

Se denota por tanto la necesidad de volver la mirada al contexto, al enfoque relacional, diferencial y de género para evitar la generalización de resultados y la definición de estigmas a grupos particulares, puesto que la misma construcción de la percepción de sí como padres o madres en condición juvenil, se produce de acuerdo a contextos históricos, culturales y sociales, con marcas propias $y$ significados para cada sujeto. El ser padres o madres en este tramo de vida, se vuelve un acontecimiento en el que deviene experiencias transformadoras para algunos sujetos ${ }^{12}$, de tal modo que, en medio de la precariedad y el desencanto, se convierte en 
un capital simbólico único que lo hace reconocido ante los demás ${ }^{3}$.

Por las razones referidas en esta introducción, se plantea en este artículo, presentar un producto que hace parte de la investigación realizada en el marco del proyecto de doctorado, denominado "experiencias de crianza de padres y jóvenes del municipio de Cúcuta"; vislumbrar el panorama histórico en el que se connota el embarazo en condición juvenil en Colombia, el cual ha sido un evento de especial atención construido a partir de la normatividad y de las prácticas sociales y culturales vigentes; posteriormente, se presenta el contexto y trayectoria del mismo en la ciudad de Cúcuta.

\section{MATERIALES Y MÉTODOS}

Con el objetivo de obtener la información se utilizaron motores de búsqueda académicos como Scirus y Google scholar, así como bases de datos: Scopus, ScienceDirect, Nursing@Ovid, Pubmed y Proquest utilizando para ello palabras clave: embarazo adolescente, trayectoria de embarazo adolescente, maternar y parternar joven, paternidad adolescente, maternidad y paternidad adolescente en Colombia y Cúcuta, en idiomas español, portugués e inglés. Se indagó además por documentos históricos sin tener en cuenta un rango de tiempo, entre ellos Planes de Desarrollo, Leyes en salud, informes y encuestas. Se obtuvieron las Actas de las mesas intersectorial municipal y departamental de prevención de embarazo en adolescentes del año 2017 y primer trimestre del año 2018, no pudiendo acceder a otras de años anteriores. Junto a estas últimas, se revisó agendas de trabajo y planes de acción propuestos por cada sector. Se analizaron además estadísticas vitales del Departamento Administrativo Nacional de Estadística (DANE) de los años 2012 al 2017 ${ }^{13-18}$, las cuales se encontraban a la fecha en estado de completitud y las Encuestas Nacional de Demografía y Salud (ENDS), de los años $2010^{19}$ y $2015^{20}$.

Teniendo en cuenta los descriptores referidos, se analizaron inicialmente tendencias conceptuales en el tema, consolidando 75 artículos e investigaciones terminadas, de enfoque cuantitativo y cualitativo en los últimos cinco años (2014- 2018), los cuales presentaban objetivo, número de participantes, intervenciones, comparaciones y resultados; estos estudios fueron realizados en diferentes países (Argentina, Estados Unidos, España, Brasil, Cuba y Uruguay). Posterior a este proceso, se realizó el filtro por país, Colombia y la ciudad de Cúcuta específicamente, obteniendo un total de 24 artículos, lo cual permitió identificar las caracterizaciones en Colombia y Cúcuta, así como los indicadores empíricos utilizados. Se excluyeron trabajos no concluidos, así como aquellos fuera de este rango de tiempo y contexto geográfico.

Para la revisión del contexto histórico en Colombia y trayectoria del embarazo en condición juvenil en la región, en total se hallaron además 33 documentos, así como 7 Actas de las mesas referidas y estadísticas del DANE desde el año 2012 a 2017. La información fue vaciada en una base de datos en Excel organizándola por tipo de documento, autores, año, objetivos, metodología, indicador empírico y hallazgos.

\section{RESULTADOS Y DISCUSIÓN}

\section{Connotación histórica del embarazo en condición juvenil en Colombia}

Puede decirse que la construcción social que se fue tornando alrededor de lo que se ha denominado embarazo en condición juvenil en Colombia, ha sido definido como un evento que desborda los límites del orden social, esto obedece a un contexto histórico permeado por algunas situaciones tanto del orden nacional como internacional. En el ámbito nacional, a partir del año 1964, se fue dando un seguimiento y vigilancia a la natalidad de cada mujer, puesto que allí emergió una "alarma" cuando se revelaron los resultados de la primera Encuesta de Fecundidad, realizada por el Centro Latinoamericano de Demografía (Celade) en siete capitales de la región. Esta encuesta demostró que, después de Ciudad de México, Bogotá tenía el promedio más alto de hijos por mujer en unión: $(5,5)$. Lo anterior, promovió a iniciar un proceso de 
regulación que el Estado debía tener sobre la población para evitar su multiplicación de manera tangencial ${ }^{21}$.

Al parecer desde esta época, la fecundidad "desproporcionada" se convierte en un asunto prioritario para el Estado, instaurándose de esta manera en el marco de los planes de desarrollo y programas estratégicos del país, una pretensión de desarraigo de esta "problemática". Una figura política influyente en este proceso fue el expresidente Alberto Lleras Camargo quien, en 1965, en la Asamblea Panamericana de Población, expresó en su discurso inaugural lo siguiente: "para quienes no queremos que la humanidad de nuestra patria se ahogue en este abismo por indiferencia y por imprevisión, la solución humana, la solución cristiana, la solución económica, la solución política, es el control de la natalidad y cuanto antes mejor" ${ }^{\prime 22}$.

De esta forma, en 1970 emergen planes de gobierno y premisas, en los que se plantea la necesidad de mantener la vigilancia sobre el crecimiento de la población, especialmente en "la ocurrencia de embarazos a temprana edad", declarando así mismo que esta situación es "una problemática social y de salud pública necesaria de ser intervenida..." como se refiere en el Conpes Social 147 de $2012^{23}$. Lo anterior puede visualizarse en el Plan de Desarrollo establecido por el entonces presidente Misael Pastrana Borrero (1970-1974), en el que propone "propender por una edad menos temprana para el matrimonio" 24 .

Frente a este tema y la connotación que va tomando el tener hijos(as) en un periodo temprano de la vida, se encuentra que, en el ámbito internacional existieron eventos que marcaron de manera importante la toma de decisiones en el Estado colombiano. Entre ellas está la Cuarta Conferencia Mundial sobre la Mujer ${ }^{25}$ de 1995 y la Conferencia de Población y Desarrollo de El Cairo ${ }^{26}, 1994$. En ellas se determina que el grupo de sujetos llamado "adolescentes", es el de mayor riesgo, agrupándolo a su vez como categoría de análisis para el diseño de políticas mundiales y toma de acciones en el ámbito de la Salud Sexual y Reproductiva ${ }^{27,28}$. por lo anterior, algunos países, entre ellos Colombia, retoman estas consideraciones para aprobar nuevas políticas públicas y diseñar lineamientos en relación con el control de la sexualidad y embarazos en este grupo de personas.

Así las cosas, se inicia en Colombia el diseño de las nuevas encuestas de Demografía y Salud en las que se aplica un cuestionario de hogares y otro de mujeres, teniendo como uno de sus objetivos hacer seguimiento a la fecundidad "de la mujer", especialmente a aquellas denominadas adolescentes; de esta manera se desarrollan las encuestas de los años 1986, 1990, 1995, 2000, 2005 y 2010 en adelante ${ }^{29}$ (en ninguna de las relacionadas se hace descripción de los hombres adolescentes padres). Se diseña, además, la política en Salud Sexual y Reproductiva publicada en $2003^{30}$ y se crean documentos y proyectos que aportan estrategias para la disminución de estos embarazos, como por ejemplo el Conpes Social 147 en el año 2012, que planteó en dirección al alcance de los Objetivos de Desarrollo del Milenio para el año 2015, "lineamientos generales para el diseño, formulación y desarrollo de una estrategia integral para reducir el embarazo en la adolescencia en Colombia" ${ }^{23}$.

Este grupo de normativas y estrategias para vigilar y controlar la fecundidad, especialmente de aquellas mujeres denominadas "madres precoces", parecen haber sido ineficaces, pues en el informe ejecutivo de Objetivos de Desarrollo del Milenio ${ }^{31}$, producido en 2015 , se halla que, del total de nacimientos en Colombia, el $23,5 \%$ pertenecen a este grupo de sujetos, lo cual es muy disímil de la meta planteada (15\%) por el Consejo Nacional de Política Económica y Social, en el documento Conpes Social 140, de $2011^{32}$.

De otra parte, en Colombia, diversos documentos han adoptado términos como pobreza transgeneracional y "trampa de la pobreza" relacionándolos con el embarazo en adolescentes, aludiendo a éste, eventos como la desescolarización, el riesgo durante la gestación para la madre, el desconocimiento de derechos, riesgos en la salud del feto y neonato, entre otros, y aún se realizan afirmaciones como que la mujer 
tiene limitantes para la formación académica necesaria y para acceder a fuentes de empleo y generación de ingreso, si presenta en gestación durante esta época de su curso de vida ${ }^{33,34}$.

Lo anterior evidencia elementos que aportan a la construcción histórica del embarazo en condición juvenil en Colombia, en la que se visibiliza de una parte, una connotación de riesgo, precariedad, necesidades no satisfechas, de incompletitud, que de cierta forma conlleva a discriminación y estigma especialmente para las mujeres, pero además como un problema de salud pública, soportado en factores biológicos y sociales, colocándolo en un orden jerárquico de alta vulnerabilidad, sujeto a protección del Estado.

No obstante, pensar en este grupo como una comunidad homogénea, impide valorar diversos factores de su presentación, puesto que los adolescentes y jóvenes presentan circunstancias diferentes y tienen necesidades diversas. Es necesario por tanto indagar en cada contexto los cambios sociodemográficos y determinantes sociales en relación al fenómeno, las múltiples razones por las que se hacen madres y padres en este grupo de edad, las cuales a nivel global se identifican como el desconocimiento de sus derechos sexuales y reproductivos, las prácticas de riesgo en la salud sexual, la presentación de este evento como la única salida de la violencia de su hogar y de las condiciones sociales adversas, o el proyecto de vida trazado sobre el embarazo durante este tramo de su trayectoria de vida como un anhelo ${ }^{34-36}$.

De esta forma, se presenta a continuación un análisis de la trayectoria del embarazo en condición juvenil en una zona fronteriza de Colombia con Venezuela, la cual ha tenido cambios de orden socioeconómico y político, a partir del cierre de frontera unilateral realizado por Venezuela en el año 2015. Esta es la ciudad de Cúcuta, en la que el impacto de la migración ha hecho cambios en los servicios de salud por aumento de la demanda, pero así mismo se ha dado apertura a programas de atención a migrantes, como por ejemplo en el área de la salud sexual y reproductiva. Veamos entonces la trayectoria y contexto de la región en la que se presenta el análisis del embrazo en condición juvenil de padres y madres.

\section{Trayectoria del embarazo en condición juvenil en la ciudad fronteriza de Cúcuta}

Es necesario hacer referencia a algunos datos de la región para ir contextualizando la problemática existente. Con respecto a la estructura socioeconómica de la ciudad, esta se construye por estratos sociales y se estima que la proporción de necesidades básicas insatisfechas es de $22,40 \%{ }^{37}$. En relación con el desarrollo de actividades, se reconoce de una parte como una zona en expansión comercial, con influjo agroindustrial y minera de su zona metropolitana, por lo que se ha logrado un avance que se visibiliza en la progresividad de los proyectos públicos y privados de algunos sectores ${ }^{38-}$ 40.

Para los jóvenes la cifra de desempleo en la ciudad supera el valor a nivel nacional (16,5\%), puesto que, de acuerdo a la información del Departamento Nacional de Planeación, para el primer semestre de 2018 era de $28,1 \%{ }^{41,42}$. Así, el panorama de desempleo e informalidad tienen una connotación en la gestación y crianza, que marca profundas implicaciones relacionados con el cuidado infantil, puesto que los trabajadores informales (mujeres $u$ hombres) no acceden a licencias por maternidad ni a otros derechos protegidos como en el empleo asalariado ${ }^{43}$.

\section{Caracterización de padres y madres en condición juvenil del municipio de Cúcuta}

Referente al contexto educativo de madres en condición juvenil en la ciudad, las cifras estadísticas presentadas por el Departamento Administrativo Nacional de Estadística (DANE) ${ }^{14-16}$ a partir del 2015, dan cuenta del nivel de estudios alcanzados por grupos de edad (Figura 1). 


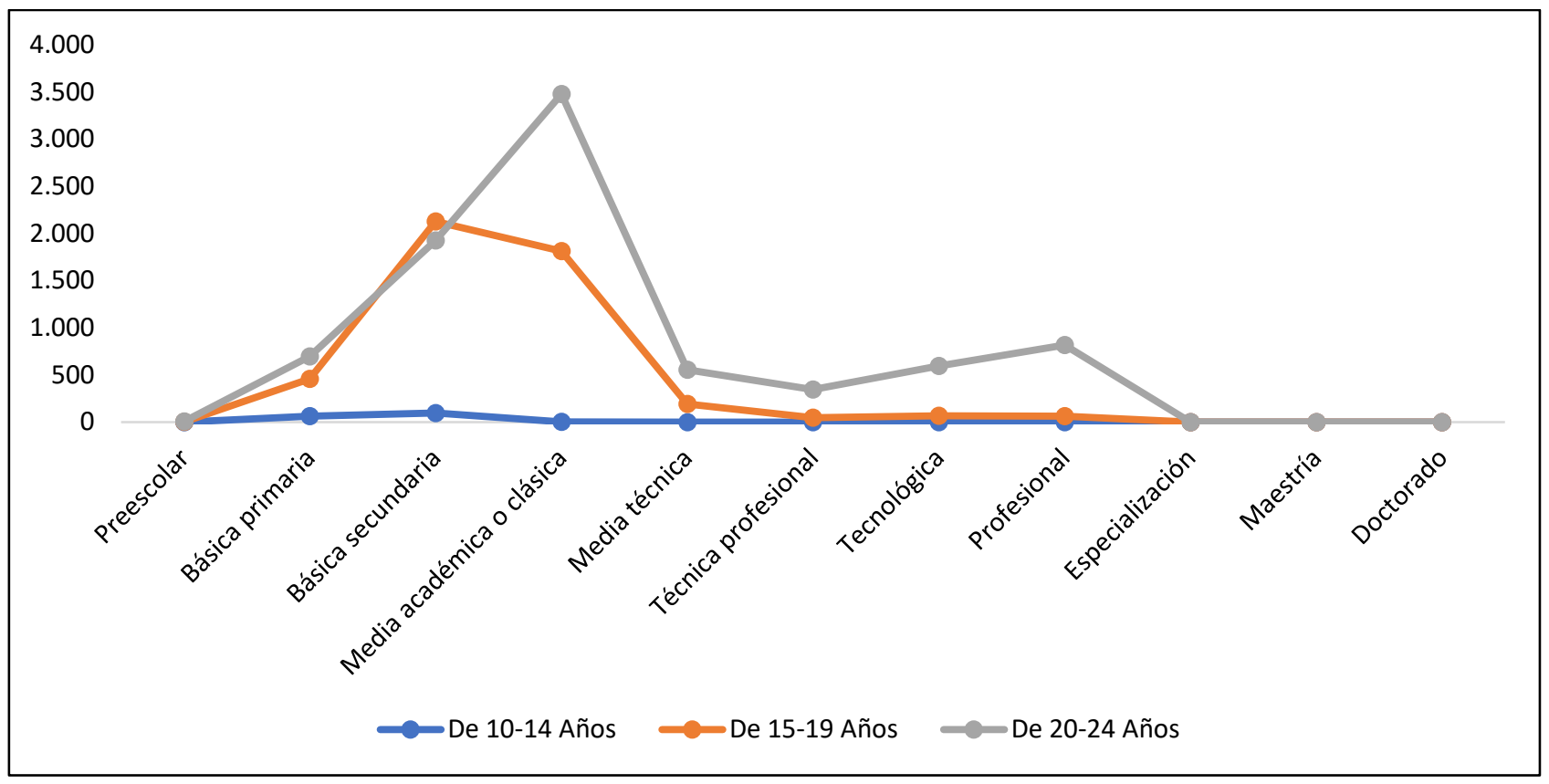

Figura 1. Nivel educativo de las madres por rangos etarios en el municipio de Cúcuta.

Inicialmente puede observarse que entre los grupos de edad durante este periodo, el de 20 a 24 años, presenta el mayor promedio de mujeres que se han convertido en madres (30,5\%), aunque el grupo de 15 a 19 años continua siendo un grupo representativo $(19,7 \%)$; en general, se observa que las mujeres de 25 a 29 años son las que han alcanzado un mayor nivel académico, obteniendo mayor promedio en el rango de educación tecnológica y profesional frente a los demás grupos. Los datos llevan a realizar otro análisis, lo cual es coherente con lo referido por la ENDS (2015), "el embarazo no es el motivo principal para suspender estudios, pues las jóvenes aluden a la situación socioeconómica como una de las razones más relevantes para hacerlo"20. En ese sentido las mujeres continuan estudios posterior a tener hijos, pero la ausencia de apoyo económico puede impedir su continuidad.

De otra parte, culturalmente esta región ha sido connotada por tener en su origen un acentuado patriarcalismo, dentro del cual, siguiendo a Gutiérrez, el hombre consideraba que "su personalidad social, vitalmente necesitaba llegar al control de esta célula social, para establecer en ella su mando, capacidad y poder" 44 . Este antecedente histórico parece permear aún las relaciones de padres y madres entre sí, así como entre ellos y sus hijos(as). Lo anterior emerge en estudios realizados en la ciudad, en los cuales se halla que asuntos como la crianza, son en su mayoría "responsabilidad" de la mujer, y que cuando las labores que integra este proceso son ejercidas por el hombre, se dan en casos de reorganización familiar, como por ejemplo por la salida de la mujer al trabajo, pudiendo tener como consecuencia, según ellos, la "afectación emocional" de este sujeto, puesto que se considera que el hombre es el proveedor económico y no el que lleva a cabo labores "hogareñas" 45,46 .

Otro aspecto relevante ha sido el rechazo que padres y madres perciben por parte de sus familias, docentes o amigos, quienes tienden a expulsarlos de sus contextos, considerándolos como un grupo que se sale de unos lineamientos establecidos en la sociedad, pues se califican como padres o madres precoces $^{47}$.

Cabe resaltar, entonces, que esta ciudad, caracterizada por grandes contrastes sociales, económicos, culturales, políticos, alberga madres y 
padres jóvenes que se desenvuelven entre los ámbitos familiares, laborales, académicos y otros espacios que emergen en su vida cotidiana de acuerdo con sus contextos específicos, en los que andan, hablan, piensan y viven la vida de manera diversa.
Con respecto al total de embarazos en condición juvenil ocurridos en el departamento Norte de Santander, la ciudad de Cúcuta aporta una gran proporción (Figura 2).

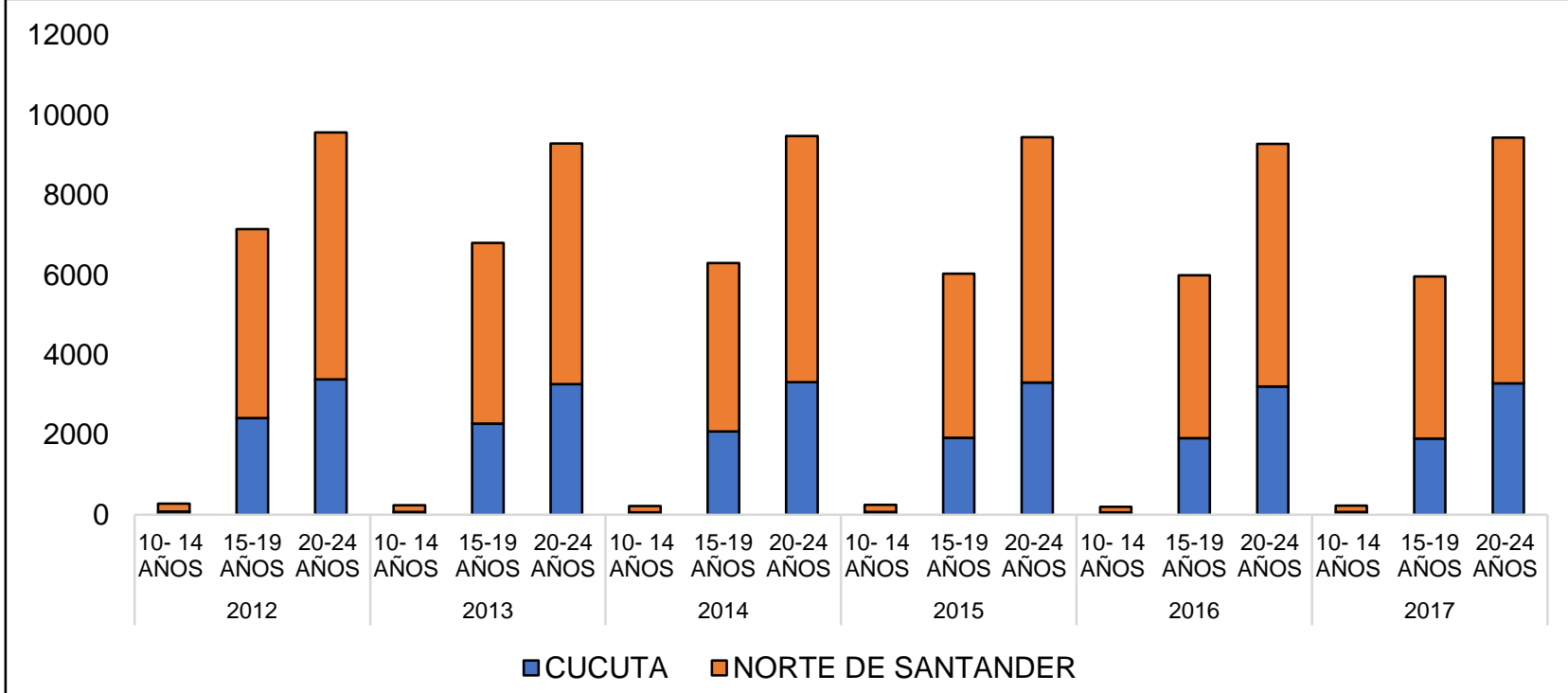

Figura 2. Proporción de embarazo juvenil en relación al total departamental 2012-2017.

Según esta información, se logra evidenciar que durante este periodo, las mujeres de la ciudad entre los 20-24 años, son las que mayormente han contribuido con el número de embarazos en el departamento, pudiendo observarse que en todos los periodos aportan más del $50 \%$ de los nacimientos, en un rango entre el $53 \%$ en el año 2016, al 55\% en el 2012, evidenciándose su descenso en el transcurso del tiempo; el grupo de mujeres de 15-19 años, muestra un descenso en la proporción de su participación dentro de los nacimientos del departamento que va del $54,7 \%$ en el año 2012 al 53,6\% en el año 2017. Igualmente llama la atención el aumento de nacimientos provenientes de madres entre 10 a 14 años, lo cual puede deberse al inicio temprano de relaciones sexuales, pero además abuso sexual y determinantes sociales que se implican en este tema en mujeres menores de 14 años, puesto que este comportamiento se asocia a determinantes de carácter estructural, como la pobreza, las inequidades sociales, las relaciones de dominación y subordinación de género y la violencia ${ }^{48}$. Se puede analizar finamente el comportamiento de este fenómeno en el municipio, como se observa en la (Figura 3). 


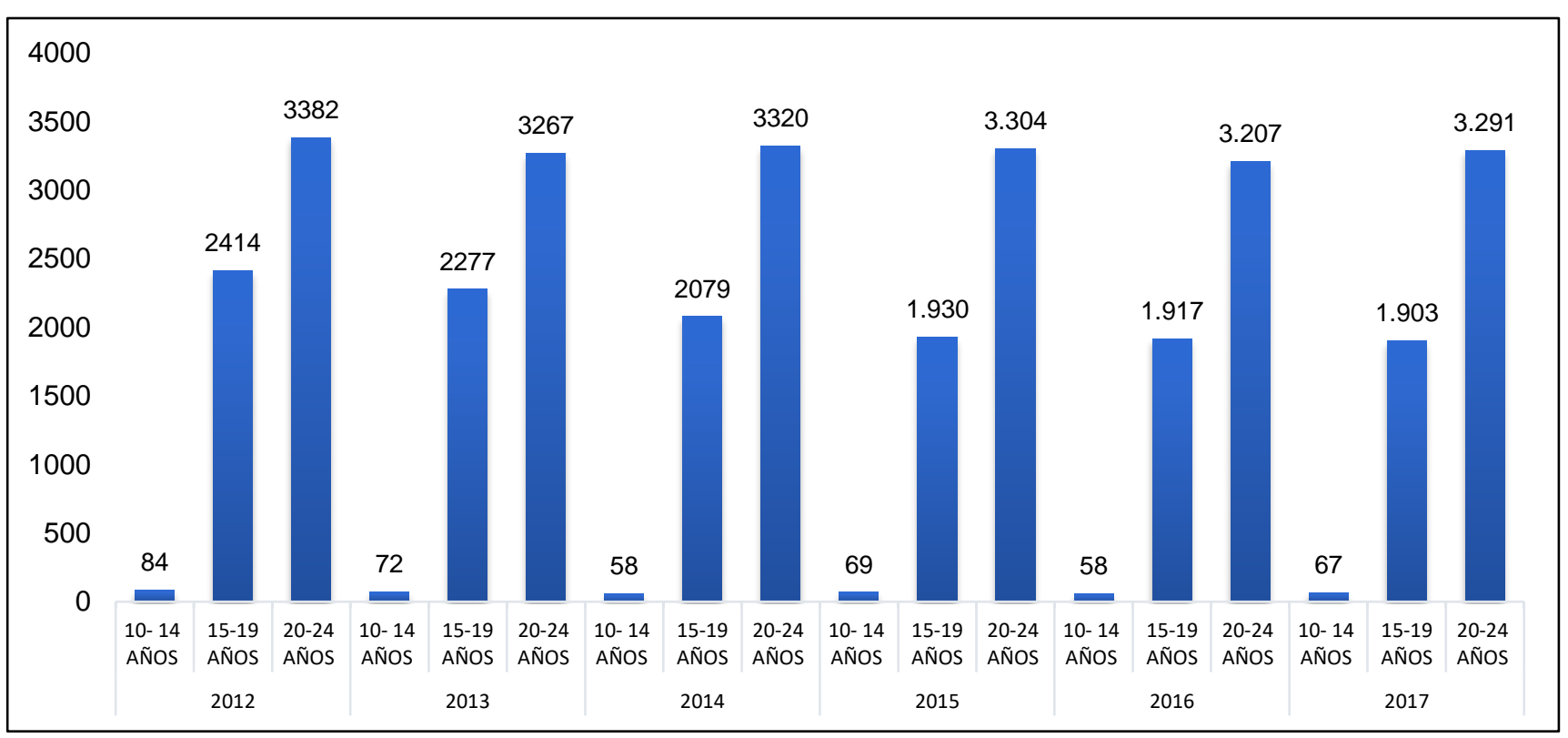

Figura 3. Trayectoria de embarazos en mujeres jóvenes de Cúcuta 2012-2017.

Al realizar la evaluación de esta información, se releva que el grupo que mayormente tiene embarazos terminados en nacimientos vivos, son mujeres de 20 a 24 años, lo cual se ha mantenido en el tiempo y permite conocer que estas mujeres, entre quienes un $27.45 \%$ tienen estudios que van de la media técnica a universitarios, deben continuar su formación profesional y requieren de apoyo familiar, del Estado y comunitario para hacerlo. Se halla además que el grupo de 15 a 19 años, ha presentado un descenso de nacimientos en este periodo. Llama la atención el número de embarazos que han ocurrido en menores de 14 años, los cuales se han mantenido proporcionalmente en el tiempo.

Estas cifras son coherentes con el comportamiento de este evento a nivel nacional, puesto que de acuerdo con los resultados de las Encuestas Nacionales de Demografía y Salud (ENDS), a partir de 2005 se confirma la tendencia decreciente del porcentaje mujeres de 15 a 19 años que ya son madres o están embarazadas de su primer hijo al pasar de $20,5 \%$ en 2005 a 19,5\% en 2010 y a $17,4 \%$ en $2015^{49}$. No obstante, para Norte de Santander, según la ENDS 2015, el porcentaje de mujeres de 15 a 19 años que ya son madres es del 15,7\%, y las que ya son madres o se encuentran embarazadas de su primer hijo a esta edad, llega al $21,4 \%$. Por lo anterior, teniendo en cuenta que el departamento se encuentra entre las 12 zonas del país con mayor índice de embarazos en jóvenes de entre los 10 y 19 años, y que la ciudad de Cúcuta aporta la mayor proporción de estos, el Instituto Colombiano de Bienestar Familiar (ICBF) la priorizó para realizar acciones de vigilancia y control sobre esta "problemática" 39 . Igualmente se estableció en el Plan Decenal de Salud Pública (PDSP) que la meta para el 2021 es reducir la cifra de embarazos en adolescentes a $22 \%{ }^{50}$.

Desde una aproximación a los estudios realizados en la ciudad con población de adolescentes y jóvenes madres, se indagó acerca de los determinantes sociales, de la salud que rodean este fenómeno. Lo anterior reconociendo la interacción de microsistemas y macrosistemas como lo plantea Bronfenbrenner ${ }^{51}$, los cuales pueden influir y afectar negativamente la autonomía y repercutir en la equidad en salud sexual y los derechos sexuales y reproductivos de la población, así como en la toma de decisiones.

Así las cosas, se retomaron entre otros, trabajos de grado y artículos del periodo de 2012 al $2017^{46,52-54}$ en los que participaban madres y padres en condición juvenil de la ciudad, hallando la siguiente 
caracterización de las mujeres; no existen amplios trabajos con hombres que permitieran analizar su trayectoria (Tabla 1).

Tabla 1. Caracterización de madres en condición juvenil en la ciudad de Cúcuta.

\begin{tabular}{|l|l|}
\hline \multicolumn{2}{|l|}{ Resultados de mayor frecuencia } \\
\hline Edad & $15-19$ años (15\%) \\
& $20-24$ años (47\%) \\
\hline Estado Civil & Unión (59\%) \\
& Solteras (23\%) \\
& Casadas (20\%) \\
\hline Nivel socioeconómico & $1(59 \%)$ \\
& $2(32 \%$ \\
\hline Escolaridad & $3(9 \%)$ \\
\hline Ocupación & Media académica (68\%) \\
& $45 \%$ Hogar; \\
& $33 \%$ informalidad \\
& $12 \%$ estudiantes \\
& $10 \%$ trabajo formal \\
\hline Tipo de familia de la que provienen & Nuclear 34\% \\
& Extensa $21 \%$ \\
\hline Acompañamiento del hombre a programas & Monoparental $5 \%$ \\
\hline de atención a la mujer & $38 \%$ en la mayoría al programa de preparación para el parto y \\
\hline
\end{tabular}

Se evidencia que dentro de las características halladas como de mayor frecuencia en el grupo de mujeres madres en condición juvenil de la ciudad, se hallan algunas que revelan las formas familiares que se producen a partir de estos casos. Como puede valorarse, el rango de edad es coherente con las cifras obtenidas del DANE en el periodo estudiado, los cuales se encuentran en los rangos de los 15 a 19 años y de 20 a 24 años. El estado civil formado en su mayoría, es la unión libre, la cual es una forma dada de manera particular en todo el país que pasó entre las mujeres de 15 a 49 años de 33,3\% en 2010 a $34,7 \%$ en $2015^{20}$.

La escolaridad de estas mujeres se encuentra en su mayor proporción en la media académica, algunas terminando su bachillerato, lo cual ha sido uno de los determinantes sociales interpersonales hallados a nivel nacional. La ocupación que realizan estas madres en su mayoría se mueve entre los oficios del hogar y la informalidad. Algunas madres relatan que deben trabajar haciendo manualidades $y$ ofreciéndolas a través de la web, vendiendo en su casa o por las calles algunos elementos de consumo diario, acompañando a su familiar en la venta de gasolina de contrabando, entre otros, lo cual ya realizaban antes de quedar embarazadas. Estas ocupaciones se encuentran muy relacionadas con el nivel socioeconómico de las madres de los estudios, puesto que en su mayoría se encuentran agrupadas en estratos bajos.

Los hallazgos presentan determinantes tanto próximos como intermedios y distales, que muestran una realidad contextualizada en la región, en el caso de mujeres en condición juvenil embarazadas o con hijos recién nacidos, sobre las cuales deviene en algunos casos el estigma y desvaloración de condición humana, por el hecho de tener hijos a esta edad.

Toda esta información ha sido un tema de análisis e intervenciones en el municipio, puesto que tanto 
desde el ente Territorial, la gobernación del departamento, la alcaldía municipal, el Instituto Colombiano de Bienestar Familiar, la academia, entre otras instituciones que tienen parte en las mesas departamentales y municipales de Infancia y Adolescencia, como desde la mesa de "prevención de embarazo en adolescentes", han trabajado en el diseño de estrategias que aporten a este grupo de mujeres y jóvenes madres.

Tanto el trabajo realizado en la mesa municipal y departamental de la ciudad, durante los últimos años, como el de la academia, secretaría de salud y Ente Territorial, ha estado enfocado en la prevención del embarazo en niñas y adolescentes; para lo cual se ha dado a conocer el estado epidemiológico y análisis situacional de la región, se actualiza en lineamientos y acciones a realizar dirigidas por el ente central, así como en el diseño de actividades interactivas con grupos de pares para los y las adolescentes en actividades concurrentes como la semana Andina.

Las mesas trabajan sobre el desarrollo de un plan de acción específico interdisciplinario, plasmando así mismo la necesidad de dar el debido seguimiento a las actividades desarrolladas y a la capacitación en rutas de atención; igualmente se han instaurado proyectos a ejecutar con alcance local y departamental. La mesa departamental, ha trabajado en varias direcciones, entre ellas promoviendo la implementación de la cátedra de educación sexual en la básica y secundaria y acordando el cumplimiento de lineamientos del orden nacional, así como el trabajo dirigido a "contener los embarazos en adolescentes hasta un $22 \%$ ", meta planteada para el departamento en el PDD 2016-2019. La academia ha realizado proyectos de extensión tanto en el área de la salud, como de la educación en escuelas y colegios públicos en temas como "cuidado a las madres gestantes y su hijo por nacer", "cuidado a la puérpera adolescente y su recién nacido y familia", escuela saludable y promoción del eje transversal en salud sexual y construcción de ciudadanía, todo lo cual se ha ido evaluando a partir de indicadores de resultado y evaluaciones de satisfacción.
Estos esfuerzos realizados en conjunto, continúan su trabajo hacia la meta propuesta; no obstante a pesar de tratar este evento como un conflicto que deba resolverse o un "problema de salud pública" que busca causalidades a través del trabajo intersectorial, las mujeres en condición juvenil de la ciudad integran la mayor proporción de madres entre la población en edad fértil, como puede valorarse en la Encuesta Nacional de Demografía y Salud de $2015^{20}$ que refiere que del total de madres en Colombia, las mujeres de la región del Norte de Santander con edades entre 15 y 19 años, ocupan un $21,4 \%$, superando valores de otras regiones como Bogotá (12,2\%), Antioquía (16,3\%), Valle (11,7\%), algunos municipios de la región Andina, entre otros. Contrario a lo sucedido en relación con los hombres que se convierten en padres en ese mismo rango de edad, de los cuales en la región solo un $0,8 \%$ ha tenido por lo menos un hijo. Se puede observar a partir de estos datos, la necesidad de repensar las estrategias educativas y asistenciales realizadas a la fecha frente al tema, y como refiere Funes "conviene que seamos conscientes de que la tensión o la frustración educativa que nos invade... debe llevarnos a descubrir que convivimos con personajes contradictorios (en permanente contradicción personal) y todo puede ser cara o cruz o las dos cosas a la vez" ${ }^{\prime \prime 5}$.

\section{CONCLUSIÓN}

La revisión realizada permite visualizar el contexto histórico en el que emerge y se ha ido hilando la concepción del embarazo en condición juvenil en Colombia; se evidencia un influjo internacional que aporta en gran manera a la perspectiva y adscripción a una visión de riesgo de este fenómeno, así como las estrategias de seguimiento y control a este grupo de sujetos.

No obstante, entendiendo que la fecundidad y el embarazo en condición juvenil es una situación relevante en la que se imbrican variables biológicas, pero además sociales como la vulnerabilidad social y discriminación, requiere ser abordada por equipos interdisciplinarios, en salud, educación, ciencias 
sociales, laborales y culturales, y así mismo se hace necesario que en la construcción de políticas públicas, se reflexione frente a preguntas como las siguientes: ¿ha sido la connotación histórica del embarazo en condición juvenil producto del análisis de los cambios sociodemográficos y necesidades de la población?, ¿han sido pertinentes los lineamientos e intervenciones establecidos para trabajar con la población en condición juvenil antes de su embarazo, durante o después de este?, pero sobre todo ¿se le ha preguntado a los y las adolescentes y jóvenes sobre sus deseos en salud sexual y reproductiva?

El análisis reflexivo de estas preguntas, permitirán ofrecer un cuidado congruente con las necesidades de la población, contribuyendo a la disminución de embarazos no deseados, a disminuir la presencia de indicadores de morbimortalidad materna $y$ perinatal; a contribuir con el diseño de estrategias y/o programas enfocados en la equidad social y de género, que apoyen a los padres y madres adolescentes para continuar sus proyectos de vida; a ofertar servicios de cuidado a los niños y niñas de padres en condición juvenil, entre otros.

Frente a la trayectoria del fenómeno y las intervenciones realizadas en la ciudad de Cúcuta, se halla que este no se ha logrado "contener", especialmente porque emergen nuevos casos en el grupo etario de menor edad, lo cual llama la atención sobre el riesgo de este grupo etario y los determinantes sociales aunados a este evento.

Lo anterior subraya la necesidad de ver estos fenómenos desde un enfoque relacional que analice el sujeto en relación con los contextos que le rodean, sean estos políticos, económicos, sociales o culturales, evitándose de esta manera la esencialización de correlaciones con enfoque unidireccional.

\section{DECLARACIÓN SOBRE CONFLICTO DE INTERESES}

La manifiesta no tener conflicto de intereses autora.

\section{REFERENCIAS BIBLIOGRAFICAS}

1. Ministerio de Salud y Protección Social y el Fondo de Poblaciones Unidas (UNFPA). Protocolo de Atención a la menor de 15 años embarazada. Bogotá: UNFPA; 2014.

2. Santillán El, González EC. Nociones de juventud: aproximaciones teóricas desde las ciencias sociales. Rev culturales. [Revista en la internet]. 2016 [Consultado 2018 octubre 15]; 4(1):113$136 . \quad$ Disponible en: http://www.scielo.org.mx/pdf/cultural/v4n1/24 48-539X-cultural-4-01-00113.pdf

3. Reguillo R. La condición juvenil en el México contemporáneo. Biografías, incertidumbre y lugares, en Reguillo, Rossana (Coord.), Los jóvenes en México, Fondo de Cultura Económica. México: Consejo Nacional para la Cultura y las Artes; 2010.

4. Vommaro P. Juventudes latinoamericanas: diversidades y desigualdades. Temas. [Revista en la Internet]. 2016 [Consultado 2018 noviembre 11]; 87:4-11. Disponible en: http://www.temas.cult.cu/sites/default/files/art iculos_academicos_en_pdf/01\%20Vommaro_0. pdf

5. Chaves M. Jóvenes, territorios y complicidades: una antropología de la juventud urbana. Buenos Aires: Espacio Editorial; 2010.

6. Organización Mundial de la Salud. Salud de la madre, el recién nacido, del niño y del adolescente. Salud del adolescente. Suiza: OMS; 2016

7. República de Colombia. Congreso de Colombia. Ley 1098 de 2006, por la cual se expide el Código de la Infancia y la Adolescencia. Diario Oficial No. 46446 (nov. 8 2006).

8. República de Colombia. Congreso de Colombia. Ley 1622 de 2013, por medio de la cual se expide el estatuto de ciudadanía juvenil y se dictan otras disposiciones. Diario Oficial No. 48776 (abril 29 2013).

9. Bulgach V, Zunana C, Califano P, Rodríguez, Mato R. Madres adolescentes internadas junto con sus hijos en un hospital de alta complejidad: diferencias entre la adolescencia temprana- 
media y tardía. Archivos Argentinos de pediatría. [Revista en la Internet]. 2018 [Consultado 2018 noviembre 11]; 116(2):160-164. Doi: https://doi.org/10.5546/aap.2018.160

10. Kirbas A, Hacer G, Daglar K. Pregnancy in adolescent: it is an obstetrical Risk?. Journal of Pediatric and Adolescent Gynecoloy. [Revista en la Internet]. 2015 [Consultado 2018 noviembre 11]; 29(4):367-371. Doi: https://doi.org/10.1016/j.jpag.2015.12.010

11. Althabe F, Moore JL, Gibbons L, Berrueta M, Goudar S, Chomba E, et al. Adverse maternal and perinatal outcomes in adolescent pregnancies: The Global Network's Maternal Newborn Health Registry study. Reproductive Health. [Revista en la Internet]. 2015 [Consultado 2018 noviembre 11]; 12(Suppl 2):2-9. Doi: https://doi.org/10.1186/1742-4755-12-S2-S8

12. Bombino Y, Quintana L. Ser madre adolescente: "una experiencia bonita, pero complicada". Estudio de casos en Barinas, 2012. Novedades en Población. [Revista en la Internet]. 2015 [Consultado 2018 noviembre 11]; 11(21):78-88. Disponible en: http://scielo.sld.cu/scielo.php?script=sci_arttext \&pid=S181740782015000100006\&lng=es\&tlng=es.

13. Departamento Nacional de Estadística. Información para todos. Nacimientos 2012. [Internet]. Bogotá: DANE [Consultado 2018 octubre 10]. Disponible en: https://www.dane.gov.co/index.php/Estadística s-por-tema/salud/nacimientos-ydefunciones/nacimientos/nacimientos-2012

14. Departamento Nacional de Estadística. Información para todos. Nacimientos 2013. [Internet]. Bogotá: DANE [Consultado 2018 octubre 10]. Disponible en: https://www.dane.gov.co/index.php/Estadística s-por-tema/salud/nacimientos-ydefunciones/nacimientos/nacimientos-2013

15. Departamento Nacional de Estadística. Información para todos. Nacimientos 2014. [Internet]. Bogotá: DANE [Consultado 2018 octubre 10]. Disponible en: https://www.dane.gov.co/index.php/Estadística s-por-tema/salud/nacimientos-ydefunciones/nacimientos/nacimientos-2014

16. Departamento Nacional de Estadística. Información para todos. Nacimientos 2015. [Internet]. Bogotá: DANE [Consultado 2018 octubre 10]. Disponible en: https://www.dane.gov.co/index.php/Estadística s-por-tema/salud/nacimientos-ydefunciones/nacimientos/nacimientos-2015

17. Departamento Nacional de Estadística. Información para todos. Nacimientos 2016. [Internet]. Bogotá: DANE [Consultado 2018 octubre 10]. Disponible en: https://www.dane.gov.co/index.php/Estadística s-por-tema/salud/nacimientos-ydefunciones/nacimientos/nacimientos-2016

18. Departamento Nacional de Estadística. Información para todos. Nacimientos 2017. [Internet]. Bogotá: DANE [Consultado 2018 octubre 10]. Disponible en: https://www.dane.gov.co/index.php/Estadística s-por-tema/salud/nacimientos-ydefunciones/nacimientos/nacimientos-2017

19. Profamilia. Encuesta Nacional de Demografía y Salud. 2010. [Internet]. Bogotá: DANE [Consultado 2018 octubre 10]. Disponible en: http://profamilia.org.co/docs/ENDS\%202010.pd $f$

20. Profamilia. Encuesta Nacional de Demografía y Salud. 2015. [Internet]. Bogotá: DANE [Consultado 2018 octubre 10]. Disponible en: http://profamilia.org.co/docs/ENDS\%20\%20TO MO\%20I.pdf

21. Dáguer C, Riccardi M. Al derecho y al revés. La revolución de los derechos sexuales y reproductivos en Colombia. Bogotá: Printex Impresores; 2005.

22. Tirado A. Los años sesenta, una revolución en la cultura. Bogotá: Debate; 2014.

23. Consejo Nacional de Política Económica y Social (CONPES), República de Colombia, Departamento de Planeación Nacional. Documento Conpes Social 147 de 2012: "Lineamentos para el desarrollo de una estrategia para la prevención del embarazo en la adolescencia y la promoción de Proyectos de vida 
para los niños, niñas y jóvenes en edades entre 6 y 19 años". Bogotá. CONPES; DNP; 2012. [Internet]. [Consultado 2018 octubre 10]. Disponible en: https://colaboracion.dnp.gov.co/CDT/Conpes/S ocial/147.pdf

24. Departamento Nacional de Planeacion. Planes de Desarrollo anteriores. Las cuatro estrategias (1970-1974) - Misael Pastrana [Internet]. [Consultado 2018 jul 5]. Disponible en: https://www.dnp.gov.co/Plan-Nacional-de-

Desarrollo/Paginas/Planes-de-Desarrolloanteriores.aspx

25. Organización de las Naciones Unidas. Informe de la Cuarta Conferencia Mundial sobre la Mujer. Beijing: 4 al 15 de septiembre de 1995. [Internet]. [Consultado 2018 jul 5]. Disponible en: http://www.equidadmujer.gov.co/ejes/Docume nts/NormativaNacional/Cuarta\%20Conferencia \%20Mundial\%20sobre\%20la\%20Mujer\%20en\%2 OBeijing\%20de\%201995.pdf

26. Organización de las Naciones Unidas. Conferencia Internacional sobre la Población y el Desarrollo. El Cairo: 5 al 13 de septiembre de 1994. [Internet]. [Consultado 2018 jul 7]. Disponible en: https://www.unfpa.org/sites/default/files/pubpdf/icpd_spa.pdf

27. Fondo de Población de las Naciones Unidas (UNFPA), Secretaría Distrital de salud. Embarazo en adolescente en Bogotá: construir nuevos sentidos y posibilidades para el ejercicio de derechos. LEGIS S.A. Bogotá: 2011.

28. Corrigan P, Sayer D. El gran arco: la formación del Estado inglés como revolución cultural. En: Lagos, María L. y Calla, Pamela. (comps.). Antropología del Estado: Dominación y prácticas contestatarias en América Latina. La Paz: INDH/PNUD; 2007.

29. Ministerio de Salud y Protección Social. Dirección de Epidemiología y Demografía. 2013. [Internet]. [Consultado 2018 julio 7]. Disponible en: https://www.minsalud.gov.co/sites/rid/Lists/Bib liotecaDigital/RIDE/VS/ED/presentacionends.pdf

30. Colombia. Ministerio de Protección Social. Política Nacional de Salud Sexual y Reproductiva.
[Internet]. Bogotá: 2003. [Consultado 2018 julio 7]. Disponible en: https://www.minsalud.gov.co/sites/rid/Lists/Bib liotecaDigital/RIDE/DE/LIBRO\%20POLITICA\%20S EXUAL\%20SEPT\%2010.pdf

31. Organización de las Naciones Unidas. Objetivos de Desarrollo del Milenio. Informe de 2015. Nueva York: ONU. [Internet] [Consultado 2018 jul 7]. Disponible en: http://www.un.org/es/millenniumgoals/pdf/20 15/mdg_2015_s_summary_web.pdf

32. Colombia. Departamento de Planeación Nacional. Documento Conpes Social 140 de 2011: "Modificación a Conpes Social 91 de 14 de junio de 2005: "Metas y estrategias de Colombia para el logro de los objetivos de Desarrollo del Milenio". Bogotá D.C.: CONPES; DNP; 2011. [Internet] [Consultado 2018 jul 7]. Disponible en: https://colaboracion.dnp.gov.co/CDT/Conpes/S ocial/140.pdf

33. Pacheco C. Agencia social, sexualidad y embarazo en menores de 15 años. Revista Gerencia y Políticas de Salud, [Revista en la Internet]. 2015 [Consultado 2018 noviembre 11];14 (29):

62-82. Doi: https://dx.doi.org/10.11144/Javeriana.rgyp s14-29.asse

34. Castillo L. Desaciertos en la prevención del embrazo en adolescentes. Salud Uninorte. [Revista en la Internet]. 2016 [Consultado 2018 jul 7]; 32(3): 543-551.. Disponible en: http://www.scielo.org.co/pdf/sun/v32n3/v32n3 a16.pdf

35. Carvajal R, Valencia $H$, Rodríguez R. Factores asociados al embarazo en adolescentes de 13 a 19 años en el municipio de Buenaventura, Colombia. Rev de la Universidad Industrial de Santander. Salud. [Revista en la Internet]. 2017 [Consultado 2018 jul 7]; 49(2): 290-300. Disponible https://www. redalyc.org/pdf/3438/3438512110 04.pdf

36. Mazuela R, Trejos A, Reyes L. Percepción del embarazo adolescente en el departamento Norte de Santander, Colombia. Rev Salud pública. [Revista en la Internet]. 2017 
[Consultado 2018 jul 7]; 19(6):733-738. Doi: https://doi.org/10.15446/rsap.V19n6.57679

37. Departamento Nacional de Estadística. Encuesta de calidad de vida. Desagregada a 30 de junio de 2012. [Internet]. Bogotá: DANE [Consultado 2018 nov 11]. Disponible en: https://www.dane.gov.co/index.php/Estadística s-por-tema/demografia-y-poblacion/censogeneral-2005-1/censo-general-2005

38. Departamento Nacional de Estadística. Encuesta Nacional de Calidad de Vida 2015. [Internet]. Bogotá: DANE [Internet]. [Consultado 2018 nov 11]. Disponible en: https://www.dane.gov.co/files/investigaciones/ condiciones_vida/calidad_vida/Presentacion_EC V_2015.pdf

39. Villamizar W. Plan de Desarrollo Departamental 2016-2019 "Un Norte productivo para todos". [Internet] Bogotá: DANE [Consultado 2018 nov 11]. Disponible en: http://www.sednortedesantander.gov.co/sitio/i mages/documentos/informesdelsector/PDD\%20 NDS\%202016-2019.pdf

40. Departamento Nacional de Estadística. Informe de Coyuntura Económica Regional 2015 [Internet] Bogotá: DANE [Consultado 2018 nov 11]. Disponible en: https://www.dane.gov.co/files/icer/2015/ICER_ Norte_de_Santander2015.pdf

41. Departamento Nacional de Estadística. Gran Encuesta Integrada de Hogares (GEiH), 2018. Boletín Técnico. [Internet] Bogotá: DANE [Consultado 2018 nov 11]. Disponible en: https://www.dane.gov.co/files/investigaciones/ boletines/ech/ech/bol_empleo_ene_18.pdf

42. Departamento Nacional de Planeación. Dirección de estudios económicos. Mercado Laboral Urbano-primer semestre de 2018 [Internet]. Bogotá: DANE [Consultado 2018 nov 11]. Disponible en: https://colaboracion.dnp.gov.co/CDT/Estudios\% 20Econmicos/7.Informe\%20MLU\%20Cucuta\%20 2018\%20IS.pdf

43. Faur E. Cuidado Infantil en el Siglo XXI. Mujeres malabaristas en una sociedad desigual. Buenos
Aires: Siglo Veintiuno Editores Argentina S.A. 2014.

44. Gutiérrez V. Familia y Cultura en Colombia. Tipologías, funciones y dinámica de la familia. Manifestaciones múltiples a través del mosaico cultural y estructuras sociales. Medellín: Editorial Universidad Antioquía; 1994.

45. Gutiérrez P, Álvarez B, Corredor MC, Martínez IC. Impacto familiar por la variabilidad laboral. Dos derechos constitucionales: trabajo y familia. Revista Academia \& Derecho. [Revista en la Internet] 2016 [Consultado 2018 nov 11]; 7(13):185-214. Disponible en: http://www.unilibrecucuta.edu.co/ojs/index.ph $\mathrm{p} /$ derecho/article/view/127/184

46. Laguado T, Lafaurie MM, Vargas LM. Experiencias de participación de los hombres en el cuidado a su pareja gestante. Duazary. [Revista en la Internet] 2019 [Consultado 2018 nov 11]; 16(1): 81-94.

Doi: https://doi.org/10.21676/2389783X.2532

47. García C, Parada D. Construcción de adolescencia: una concepción histórica y social inserta en las políticas públicas. Universitas Humanística. [Revista en la Internet] 2018 [Consultado 2018 nov 11]; 85: 347-373. Doi: https://doi.org/doi:10.11144/Javeriana.uh85.ca ch

48. Colombia. Ministerio de Salud y Protección Social. Observatorio Nacional e intersectorial del embarazo adolescente. Guía Metodológica. Bogotá: Minsalud; 2013. [Internet] [Consultado 2018 nov 11]. Disponible en: https://www.minsalud.gov.co/sites/rid/Lists/Bib liotecaDigital/RIDE/VS/ED/GCFI/ross-embarazoadolescentes-oniea.pdf

49. Profamilia \& PLAN. Determinantes del embarazo en adolescentes en Colombia. Bogotá: 2018.

50. Ministerio de Salud y Protección Social. Plan Decenal de Salud Pública 2012-2021. Cúcuta: Minsalud; 2016. [Internet] [Consultado 2018 nov 11]. Disponible en: https://www.minsalud.gov.co/Documentos\%20 y\%20Publicaciones/Plan\%20Decenal\%20- 
\%20Documento\%20en\%20consulta\%20para\%20 aprobaci\%C3\%B3n.pdf

51. Bronfenbrenner U. Contextos de crianza del niño. Problemas y prospectiva. Journal for the Study of Education and Developmen [Revista en Internet] 1985 [Consultado 2018 nov 11]; 29:45$55 . \quad$ Disponible en: https://dialnet.unirioja.es/servlet/articulo?codig $o=668368$

52. Fossi JA., Zarazo HA. Efectos de la Ley 1098 del 8 de noviembre de 2006 sobre la población adolescente embrazada en el municipio de San José de Cúcuta. Trabajo de grado. Cúcuta. Universidad Libre de Colombia; 2016

53. Álvarez DF, Pino LA, Medina MA, Rodríguez YS, Uribe Á. Impacto clínico y epidemiológico de la preeclampsia y eclampsia en mujeres adolescentes primigestantes que ingresan al HUEM de la ciudad de Cúcuta. Rev IMBION [Revista en la Internet]. 2016 [Citado 08/11/18]; 3:50-55. Disponible en: http://ojs.unipamplona.edu.co/ojs_viceinves/in dex.php/INBIOM/article/download/2243/1082

54. Paredes JA, Rondón YE. Programa de cuidado en casa las puérperas y su recién nacido hasta los seis meses de edad egresados de la IPS Puente Barco Leones de Cúcuta. Trabajo de grado. Cúcuta. Universidad Francisco de Paula Santander; 2017

55. Funes M. Quiéreme cuando menos lo merezca... porque es cuando más lo necesito. España: Grupo Planeta Edición Kindle; 2018. 